\title{
L-Arginin: essenziell für die Gefäßgesundheit
}

\section{Der physiologische Schutz vor Arteriosklerose, Herzinfarkt und Schlaganfall}

\author{
Horst Robenek, Burkhard Poeggeler
}

\section{Schlüsselwörter}

Blutgefäße, Endothel, Stickstoffmonoxid (NO), L-Arginin, Herz-Kreislauf-Erkrankungen, Arteriosklerose, oxidativer Stress, Mitochondrien, asymmetrisches Dimethylarginin (ADMA)

\section{Zusammenfassung}

Aktuelle Studien belegen die entscheidende Rolle der Aminosäure L-Arginin und des daraus gebildeten Botenstoffs Stickstoffmonoxid (NO) für die Gefäßgesundheit. Eine ausreichende Versorgung beugt aufgrund der vasoprotektiven und antioxidativen Wirkungen Bluthochdruck, Blutgerinnseln und Arteriosklerose sowie den Folgeerkrankungen wie Herzinfarkt und Schlaganfall vor.

Der menschliche Blutkreislauf ist ein Wunderwerk, das aus über 100000 km langen Röhren und Gefäßen sowie dem Herz als Motor besteht, mit dem täglich mehr als 7000 Liter Blut durch unseren Körper gepumpt werden [1]. Über das Blut werden unsere Organe mit Sauerstoff und Nährstoffen versorgt. Eine gute Durchblutung unserer Gefäße ist daher die Voraussetzung für ein gesundes Leben. Die Blutgefäße sind von einer Wand umgeben, die aus drei Schichten besteht. Außen ist das Gefäß von Bindegewebe umgeben, darauf folgt eine Muskelschicht, die den Blutdruck reguliert. Innen ist das Gefäß lückenlos mit einem einschichtigen Endothel ausgekleidet, das die physiologische Barriere zwischen dem Blut und der Gefäßwand darstellt. Das Gefäßendothel ist das größte Organ unseres Körpers [2]. Würde man es ausbreiten, könnte man zwei

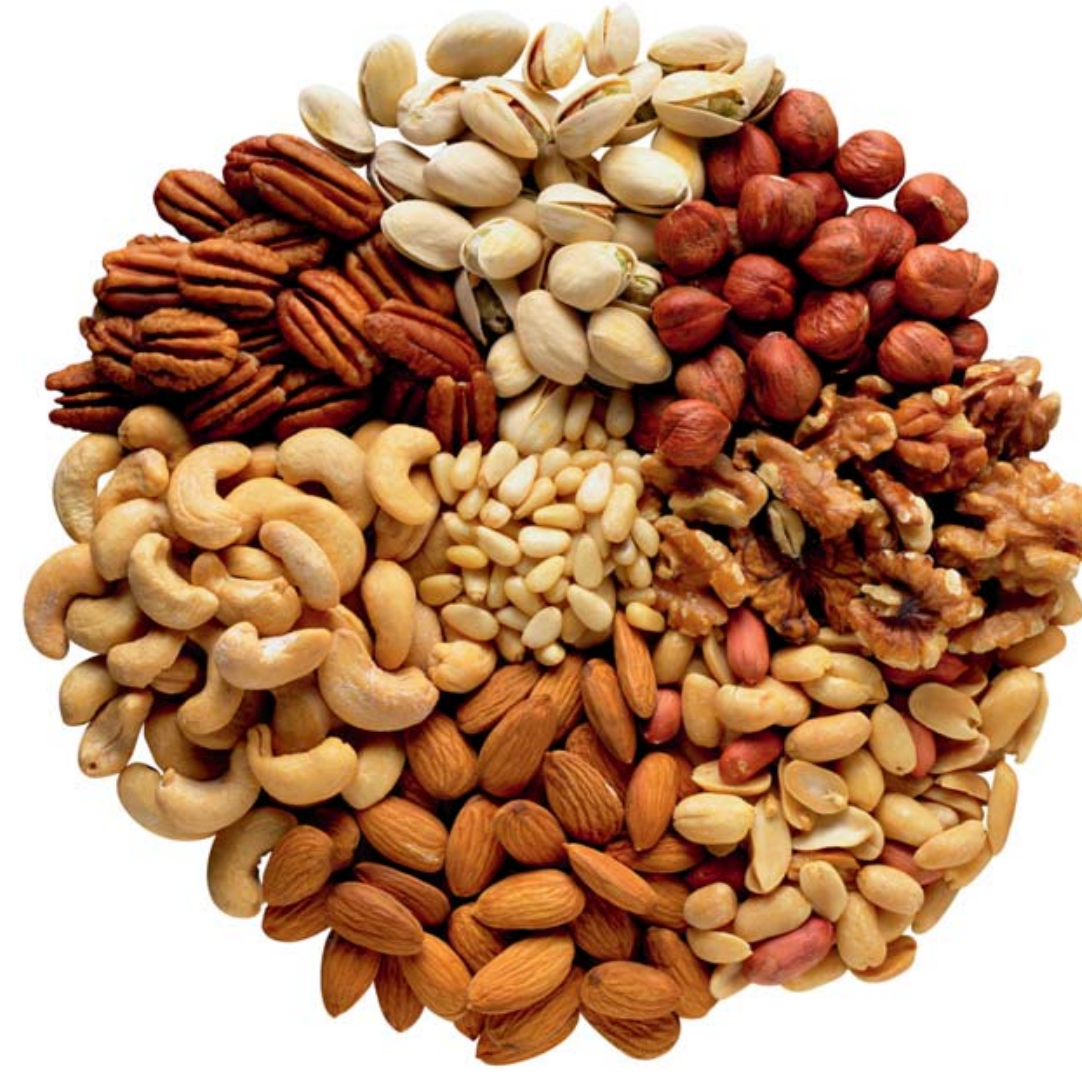

Nüsse gehören zu den Nahrungsmitteln, die besonders viel Arginin enthalten. @ PhotoDisc.

bis drei Fußballfelder damit bedecken. Sein Gewicht beträgt bis zu $2 \mathrm{~kg}$.

\section{Gefäßgesundheit ist entscheidend}

Bluthochdruck, Blutgerinnsel und Arteriosklerose mit ihren klinischen Konsequenzen wie Herzinfarkt, Schlaganfall und periphere arterielle Verschlusskrankheiten sind die Folgen eines gestörten Zusammenspiels zwischen dem
Gefäßendothel und den verschiedenen Komponenten des Blutes. Aus pathophysiologischen Untersuchungen in den vergangenen Jahren ist deutlich geworden, dass dem Gefäßendothel aufgrund der Freisetzung von Stickstoffmonoxid (NO) eine zentrale Rolle in der Gefäßgesundheit zukommt [3]. NO wird im Endothel der Blutgefäße durch die Aktivität des Enzyms NO-Synthase aus L-Arginin gebildet. 
Endotheliales NO sorgt für die Gefäßgesundheit und kann daher in physiologischer Weise der Entstehung von Bluthochdruck, Blutgerinnseln und Arteriosklerose entgegen wirken.

Heute gehört es für jeden Medizinstudenten zum Lehrbuchwissen, dass die L-Arginin-abhängige NO-Bildung bei nahezu jeder Art von Herz-Kreislauf-Erkrankung gestört ist. Die Arbeiten der Mediziner Furchgott, Murad und Ignarro dazu sind 1998 mit dem Nobelpreis ausgezeichnet worden. Tausende weitere Studien und mehrere Metaanalysen haben seither die essenzielle Wirkung von L-Arginin und NO als Botenstoff im Herz-Kreislauf-System bestätigt. Die Bedeutung dieser endogenen Moleküle für die Gesunderhaltung der Blutgefäße und als wichtiger Schutzmechanismus vor Arteriosklerose und ihren Folgeerkrankungen wie Herzinfarkt und Schlaganfall ist damit zunehmend offensichtlich geworden [3].

\section{Arteriosklerose physiologisch behandeln mit L-Arginin}

NO entspannt und weitet die Arterien, ermöglicht so einen optimalen Blutfluss und bildet die Grundlage für einen reibungslosen Sauerstoff- und Nährstofftransport zu den einzelnen Organen. Eine Funktionsstörung des Endothels führt zu einem Mangel an NO mit der Folge einer endothelialen Dysfunktion sowie Entstehung und Fortschreiten einer Arteriosklerose, was schließlich zu Komplikationen wie Herzinfarkt und Schlaganfall führen kann. Der Arteriosklerose geht also immer eine Funktionsstörung des Gefäßendothels voraus.

Die Arteriosklerose entwickelt sich zunächst unbemerkt und wird oft erst entdeckt, wenn sie weit fortgeschritten ist und bereits zu einem erhöhten Blutdruck und/oder Durchblutungsstörungen geführt hat [2]. Daher galt die Arteriosklerose lange Zeit als schicksalsbe-

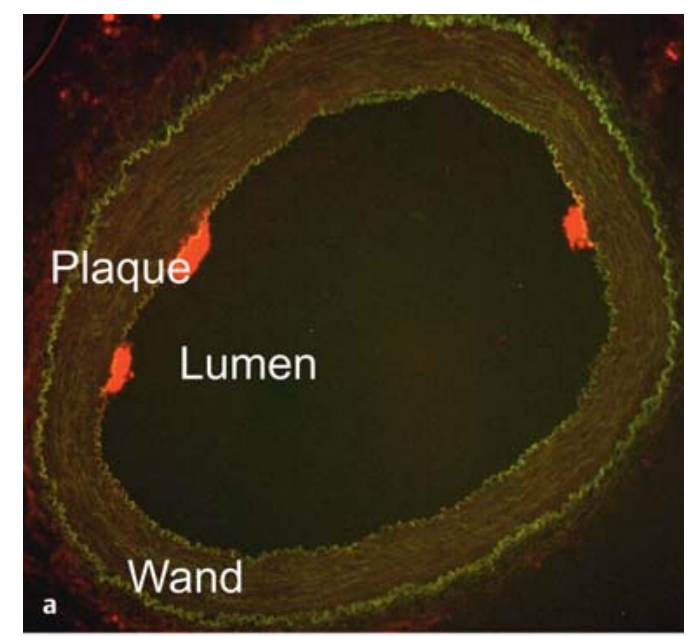

Abb. $\mathbf{1}$ a bis c Arterien mit unterschiedlichen Stadien arteriosklerotischer Plaques nach Ölrotfärbung. a kleine arteriosklerotische Plaques am Beginn ihrer Entwicklung; Arterienlumen noch nicht eingeengt; b mittelgradig fortgeschrittene Plaques; Lumen wird zunehmend kleiner; c weit fortgeschrittene Plaques; Lumen weitgehend verschlossen
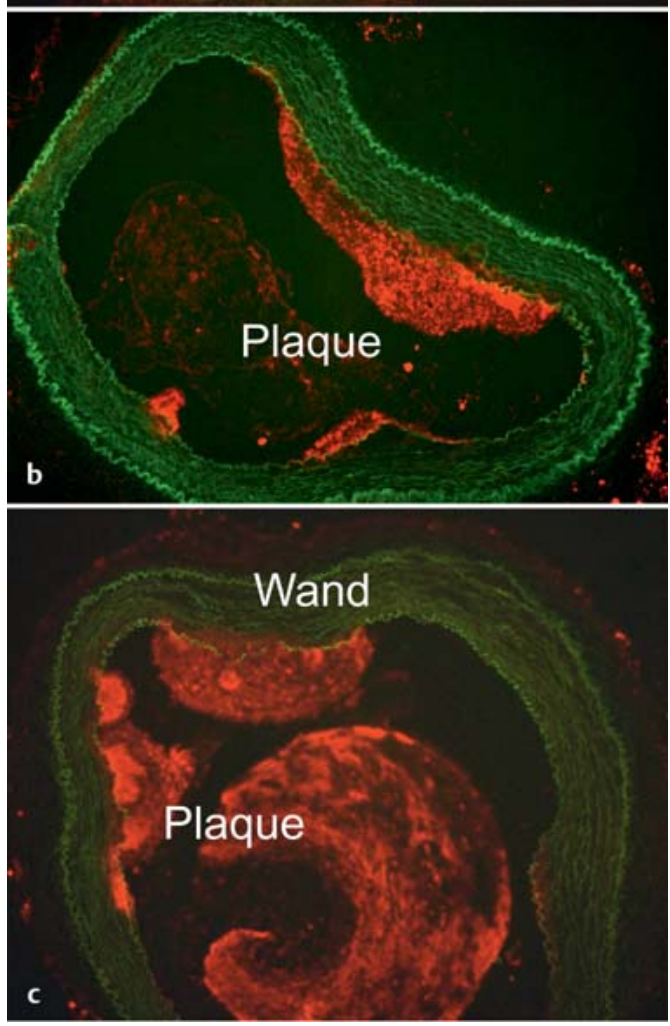

dingte, irreversible und nicht behandelbare Erkrankung.

Neuere Untersuchungen zeigen nun erstmals, dass die Gesundheit des Endothels durch die Aminosäure L-Arginin erhalten und sogar verbessert werden kann.

Dies wurde eindrucksvoll in der wegweisenden Metaanalyse von Bai et al. zur Wirkung von L-Arginin auf die Endothelzellen und deren Funktion für die Gefäßgesundheit demonstriert [4]. Für diese Analyse wurden 1466 Publikationen ausgewertet. Nur die besten Studien mit insgesamt 492 Patienten, die stringente evidenzbasierte Kriterien erfüllt haben, wurden in die engere Bewertung einbezogen. Die Verbesserung einer endothelialen Dysfunktion nach Supplementierung von L-Arginin war hochsignifikant $(\mathrm{p}=0,002)$. Bereits moderate, diätetische Mengen der Aminosäure reichen aus, um diesen positiven 
Effekt auf die Gefäßgesundheit zu erzielen.

Die essenzielle Notwendigkeit einer antiarteriosklerotischen und antithrombotischen Behandlung demonstriert $\rightarrow$ Abb. 1, die den Verlauf einer arteriosklerotischen Plaquebildung bei unbehandelten Patienten dokumentiert.

In einer weiteren Metaanalyse von Dong et al. aus dem Jahr 2011 wurde nun auch ein konsistenter und hochsignifikanter Effekt von L-Arginin auf den diastolischen und systolischen Blutdruck nachgewiesen (Senkung des systolischen Blutdrucks um 5,39 mmHg und des diastolischen Blutdrucks um 2,66 mmHg im Mittelwert bei den 387 Probanden und Patienten der eingeschlossenen Studien mit $\mathrm{p}<0,001$ ) [5].

\section{Oxidativer Stress schädigt die Gefäße}

Eine zu starke Bildung oder gestörte Entgiftung von reaktiven Verbindungen und freien Radikalen kann zu oxidativem Stress führen, der die Gefäße und v.a. das Endothel irreversibel schädigt [6]. Dieser Stress kann gefährliche Folgen für das Gefäßsystem entfalten und durch eine Oxidation seiner Moleküle und Zerstörung der Mitochondrien den Körper und seine Zellen irreversibel schädigen. Diesen degenerativen Prozessen gilt es durch entsprechende Maßnahmen präventiv und regenerativ entgegen zu wirken.

Oxidativer Stress führt

nu einer verstärkten Endothelzelldegeneration,

n zum Verlust von Telomeraseaktivität und

nu einer Telomerverkürzung,

- kurz gesagt zu all jenen Faktoren, die mit einer vorzeitigen Alterung des Gefäßsystems einhergehen [6-11], den Blutkreislauf stören und im Extremfall sogar ganz zum Erliegen bringen [6]. Da es immer ganz entscheidend auf das physiologische Gleichgewicht und die endogene Balance zwischen dem protektiven NO und endogenen gefährlichen freien Radikalen ankommt [7,9, 12], begünstigt jede Störung dieser Balance das Fortschreiten der Arteriosklerose. Diese ist stets mit einem erheblichen oxidativen Stress und damit einem schrittweisen Zusammenbruch des mitochondrialen Energiestoffwechsels verbunden [6,9].

Der oxidative Stress beruht v.a. auf der Bildung von hochreaktiven Sauerstoff- und Stickstoffverbindungen. Diese reaktiven und damit giftigen Verbindungen gehen fast alle aus den mitochondrialen Stoffwechselreaktionen im Endothel hervor $[6,9]$. Bei unzureichender Entgiftung durch endogene protektive Mechanismen kann daher dieser oxidative Stress dem Organismus gefährlich werden.

Die Hemmung der Bildung von endothelialem NO durch oxidativen Stress [6,7, 10,11] kann die Gefäßgesundheit so stark einschränken, dass es zu schweren Stoffwechselstörungen mit degenerativen Erkrankungen wie der Arteriosklerose und den damit immer verbundenen schwerwiegenden Durchblutungsstörungen kommt.

\section{Schutzfaktor L-Arginin}

L-Arginin ist das entscheidende und alleinige endogene Substrat für die Bildung von NO. Bei einem Mangel an LArginin können die Enzyme, die NO bilden, entkoppelt werden und statt des protektiven NO die gefährlichen Superoxidanionradikale produzieren $[7,10$, 13]. Dies führt zu einem gefährlichen oxidativen Stress [13]. Eine Supplementation mit L-Arginin kann dem vorbeugen und so den Organismus wirksam schützen [3,6-8,10,13]. Umgekehrt führt oxidativer Stress zu einem Arginin- und NO-Mangel [7,10,11]. Diese experimentellen und klinischen Studien belegen eindrucksvoll, dass der Ernährungsbaustein L-Arginin vor oxidativem Stress schützen kann.

\section{Mitochondriale Stimulation}

Neben seiner bekannten Rolle in der Gefäßdilatation und Endothelregulation spielt L-Arginin als Vorstufe und Nährstoff mit NO als entscheidendem Botenstoff auch eine essenzielle Rolle bei der Stimulation der Aktivität und Bildung von Mitochondrien [8,14].

NO ist der bestimmende endogene Regulator der mitochondrialen Biogenese und Physiologie [14]. Damit reguliert die Versorgung mit L-Arginin Struktur und Funktion dieser endogenen Zellkraftwerke und somit auch die gesamte Energiestoffwechseleffizienz im Organismus [8].

Daher spielt die bioenergetische Stimulation durch L-Arginin als Nährstoff und Vorstufe von NO eine entscheidende Rolle als gesundheitsfördernde und -erhaltende Nahrungsaminosäure im Organismus.

\section{Mortalitätsfaktor ADMA}

Das asymmetrische Dimethylarginin (ADMA) ist der endogene Gegenspieler von Arginin. Seine Konzentrationen sind bei fast allen Personen mit Herz-Kreislauf-Erkrankungen und Stoffwechselproblemen stark erhöht. ADMA hemmt die Enzyme, welche NO bilden und verringert die Bioverfügbarkeit von L-Arginin. Erhöhte ADMA-Spiegel steigern den Bedarf an L-Arginin daher erheblich, vermindern kompetitiv die argininabhängige NO-Synthese und verursachen so einen sehr gefährlichen NOMangel, der immer mit einem erhöhten oxidativen Stress unter Bildung hochreaktiver und zerstörerischer Radikale verbunden ist. Ferner entkoppelt ADMA die Enzyme, die NO bilden. Es kommt stattdessen zu einer vermehrten Bildung der schädlichen Superoxidanionradikale $[7,10,13]$, die stark prooxidativ, proentzündlich und gefäßschädigend wirken [3]. Schließlich verbrauchen diese reaktiven Superoxide den Radikal- 
fänger NO [13] und führen so zu einer weiteren Konzentrationsabnahme dieses für die Gefäßgesundheit so essenziellen, da antioxidativ und somit vasoprotektiv wirkenden, antiarteriosklerotischen Wirkstoffs.

\section{Fazit}

Die Verfügbarkeit der Nahrungsaminosäure L-Arginin ist von entscheidender Bedeutung für die Vermeidung von oxidativem Stress, der durch ein Ungleichgewicht zwischen protektiven Radikalen wie dem NO und gefährlichen Radikalen wie den Superoxidanionradikalen verursacht wird. Da NO in der Reaktion mit überschüssigen Superoxidanionradikalen verbraucht wird, steht dieser wichtige Botenstoff nicht mehr ausreichend zur Verfügung, um eine ausreichende Durchblutung und damit eine hohe Stoffwechseleffizienz zu gewährleisten.

Der durch oxidativen Stress ausgelöste Mangel an dem Eiweißbaustein LArginin und dem protektiven Radikal NO führt daher zu einer erheblichen Beeinträchtigung wichtiger Strukturen und Funktionen des Endothels. Tausende Originalarbeiten und die entscheidenden 3 kürzlich veröffentlichten großen Metaanalysen haben ergeben, dass L-Arginin als sehr gut verträglicher natürlicher Baustein unserer Nahrung die Endothelgesundheit verbessern, den Bluthochdruck wirksam normalisieren und die Gesundheit, insbesondere bei Stoffwechselstörungen wie Diabetes mellitus, entscheidend verbessern kann.

L-Arginin wird zurzeit intensiv beforscht als ein sehr vielversprechender natürlicher Wirkstoff zur Erhaltung oder Wiederherstellung der Gesundheit des Gefäßsystems, welches alle Organe des Körpers mit Sauerstoff und Nährstoffen versorgt. Die Aminosäure kann in Zukunft in den verschiedensten Anwendungsbereichen eingesetzt werden und dürfte deshalb auch mehr und mehr in den Standardtherapien der Arteriosklerose und des Bluthochdrucks sowie in der Behandlung verschiedener Durchblutungsstörungen wie der vaskulären Demenz Einzug halten. Erste LArginin-Produkte, wie Telcor Arginin Plus und Pascovasan, werden bereits therapeutisch mit großem Erfolg in verschiedenen Indikationen eingesetzt. Das Potenzial von L-Arginin gilt es nun voll $\mathrm{zu}$ erschließen und nutzbringend für die Patienten einzusetzen.

Über die therapeutische Anwendung und die empfohlenen Verzehrsmengen informiert der Beitrag der Autoren in der Rubrik Praxis.

Interessenkonflikte: Herr Prof. Dr. Horst Robenek hat keine Interessenkonflikte. Herr PD Dr. Burkhard Poeggeler arbeitet auch für QUIRIS Healthcare, Anbieter von diätetischen Argininprodukten. Diese wirtschaftliche Verbindung wird deshalb hier ausdrücklich angezeigt.

\section{( Literatur}

[1] Ranft JM: Der menschliche Blutkreislauf ein Wunderwerk aus kilometerlangen Röhren. Im Internet: www.angiologie-online.de/ blutkrei.htm; Stand: 26.10.2012

[2] Ranft JM: Forum Gefäßmedizin. Im Internet: www.gefaess-medizin.de; Stand: 26. 10.2012

[3] Ströhle A, Hahn A: Arginin bei Atherosklerose. Deutsche Apotheker Zeitung 2012; 20 : 97-102 und 21: 74-83

[4] Bai Y, Sun L, Yang T et al. Increase in fasting vascular endothelial function after shortterm oral L-arginine is effective when baseline flow-mediated dilation is low: a meta- analysis of randomized controlled trials. Am J Clin Nutr 2009; 89: 77-84

[5] Dong JY, Qin LQ, Zhang Z et al. Effect of oral L-arginine supplementation on blood pressure: a meta-analysis of randomized, double-blind, placebo-controlled trials. Am Heart J 2011; 162: 959-965

[6] Schulz E, Gori T, Münzel T: Oxidative stress and endothelial dysfunction in hypertension. Hypertens Res 2011; 34: 665-673

[7] Förstermann $\mathrm{U}$, Li H: Therapeutic effect of enhancing endothelial nitric oxide synthase (eNOS) expression and preventing eNOS uncoupling. Br J Pharmacol 2011; 164: 213223

[8] Jobgen WS, Fred SK, Fu W] et al. Regulatory role for the arginine-nitric oxide pathway in metabolism of energy substrates. J Nutr Biochem 2006; 17: 571-588

[9] Pak YK, Jeong JH: Mitochondria: the secret chamber of therapeutic targets for age-associated degenerative diseases. Biomol Ther 2010; 18: 235-245

[10] Seljeflot I, Nilsson BB, Westheim AS et al. The L-arginine-asymmetric dimethylarginine ratio is strongly related to the severity of chronic heart failure. No effects of exercise training. J Cardiac Fail 2011; 17: 135142

[11] Venardos K, Zhang WZ, Lang C, Kaye DM: Effect of peroxynitrite on endothelial L-arginine transport and metabolism. Int J Biochem Cell Biol 2009; 41: 2522-2527

[12] Squadrito GL, Pryor WA: Oxidative chemistry of nitric oxide: the roles of superoxide, peroxynitrite and carbon dioxide. Free Radic Biol Med 1998; 25: 392-403

[13] Poeggeler B: L-Arginin schützt vor nitrosativem Stress. Stickstoffmonoxid als endogener Regulator des nitrosativen Stoffwechsels. Perfusion 2012; 25: 40-43

[14] Nisoli E, Clementi E, Paolucci C et al. Mitochondrial biogenesis in mammals: the role of endogenous nitric oxide. Science 2003; 299: 896-899

online: http://dx.doi.org/10.1055/s-0033-1350779 


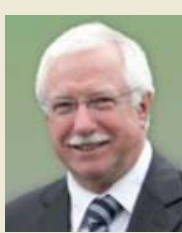
Universitätsklinikum

Münster

Albert-Schweitzer-

Campus 1

Domagkstraße 3

48149 Münster

E-Mail: robenek@

uni-muenster.de

Prof. Dr. Horst Robenek war von 1987 bis 1989 Heisenberg-Professor der Deutschen Forschungsgemeinschaft. 1989 wurde er als Universitäts-Professor an das Leibniz-Institut für Arterioskleroseforschung berufen und wurde zum Leiter des Departments für Zellbiologie und Ultrastrukturforschung bestellt. Von 1994 bis 1999 war Prof. Robenek Präsident der Deutschen Gesellschaft für Zellbiologie, 2007 wurde er zum Direktor des Leibniz-Instituts für Arterioskleroseforschung ernannt. Seit seiner Emeritierung Anfang 2012 ist er Senior-Professor an der Medizinischen Fakultät der Westfälischen Wilhelms-Universität in Münster. Seine Forschungsschwerpunkte sind die intrazellulären Vorgänge des Fettstoffwechsels unter besonderer Berücksichtigung der Speicherung von Fetten in vaskulären Zellen bei der Arteriosklerose.

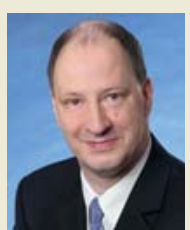

PD Dr. Burkhard Poeggeler

Eisenacher Str. 7 37085 Göttingen E-Mail: bpoegge@ gwdg.de

PD Dr. Burkhard Poeggeler wurde 1998 an die University of South Alabama in Mobile/ Alabama zum Professor für Pathologie berufen und zum Direktor des Instituts für molekulare Neuropathologie am assoziierten Mobile Medical Hospital Center ernannt. Er habilitierte 2006 an der Georg-August-Universität Göttingen und arbeitet seitdem als Privatdozent am Goettingen Research Campus und als Hochschullehrer an der Fakultät für Biologie und Psychologie der Universität Göttingen. Dr. Poeggeler entdeckte die Elektronenübertragung als entscheidenden Mechanismus der Radikalentgiftung und hat das SI-System zur Radikaldetoxifikation mitbegründet. 